\title{
Sensación de mareo y episodio de síncope
}

\author{
Clares Montón P. ${ }^{1}$, Palazuelos Molinero J. ${ }^{2}$, Sáenz Casco LV. ${ }^{3}$, Méndez Fernández M. ${ }^{4}$
}

Sanid. mil. 2013; 69 (4): 274-275; ISSN: 1887-8571

Caso clínico: Mujer de 78 años con antecedentes personales de HTA, dislipemia, insuficiencia cardíaca crónica, fibrilación auricular paroxística anticoagulada y en tratamiento con amiodarona e intervenida quirúrgicamente por doble lesión mitral e insuficiencia tricuspíde severa mediante la colocación de prótesis metálica en posición mitral, anuloplastia tricuspídea y ablación de venas pulmonares.

Acude al servicio de urgencias tres meses después de su intervención por mareos y un episodio de síncope. A su llegada la paciente presenta TA 95/55 mm Hg, FC $52 \mathrm{lpm}$, se encuentra colaboradora y en el ECG se objetiva bradicardia sinusal con prolongación de intervalo QT (600 ms) (QTc $510 \mathrm{~ms}$ ) y alternancia eléctrica de la polaridad de la onda T (fig. 1).

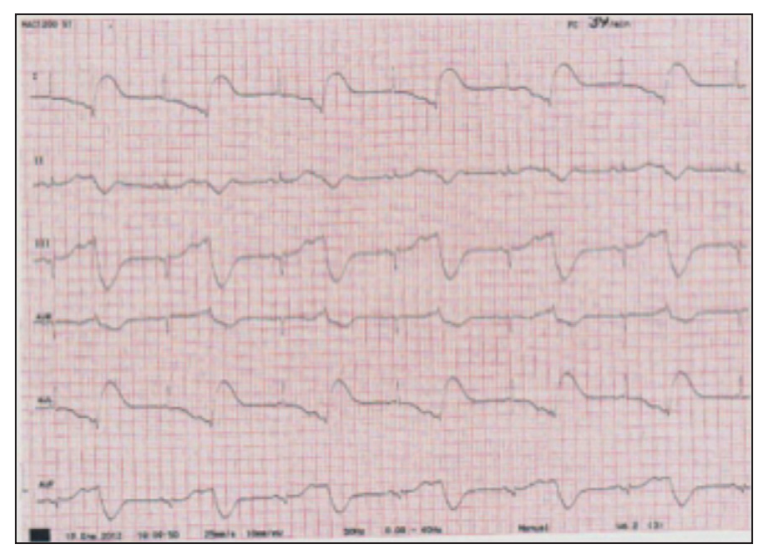

Figura 1. Bradicardia sinusal con intervalo QT prolongado (QTc $510 \mathrm{~ms}$ ).

En ECG sucesivos se observa taquicardia ventricular con QRS ancho con cambio de eje eléctrico (fig. 2A). Se realiza ecocardiograma transtorácico pórtatil que descarta cardiopatía estructural. Presenta un nuevo episodio sincopal acompañado de inestabilidad hemodinámica y pérdida del nivel de conciencia y se objetiva en el ECG taquicardia ventricular con QRS ancho a 208 1pm que precisó cardioversión eléctrica. Tras su resolución se aprecia bradicardia extrema a $25 \mathrm{lpm}$ por lo que se administra atropina y adrenalina (fig. 2B). Debido a la mala respuesta a tratamiento administrado se decide traslado a la Unidad Coronaria donde nuevamente se administran atropina y 3 gramos de sulfato de magnesio.

\footnotetext{
Médico Residente. Servicio de Cardiología.

${ }^{2}$ Médico adjunto. Servicio de Cardiología.

${ }^{3}$ Cte. Médico. Servicio de Medicina Intensiva.

${ }^{4}$ Col. Médico. Servicio de Cardiología.

Hospital Central de la Defensa Gómez Ulla. Madrid. España.
}

Dirección para correspondencia: Dra. Patricia Clares Montón. Servicio de Cardiología. Hospital Central de la Defensa Gómez Ulla. Glorieta del Ejército s/n. 28047. Madrid. patri_clares@hotmail.com

Recibido: 25 de marzo de 2013

Aceptado: 20 de junio de 2013

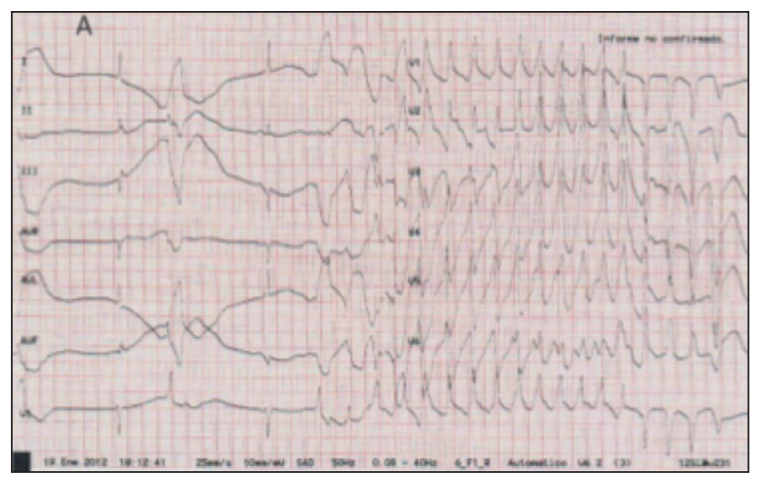

Figura 2A. Inicio de taquicardia ventricular con cambio de eje eléctrico.

Debido a la inestabilidad hemodinámica y eléctrica se inicia infusión con isoproterenol y se coloca un marcapasos transitorio vía yugular derecha. En controles analíticos no se observan alteraciones hidroelectrolíticas.

Revisado el tratamiento farmacológico habitual, la familia nos informa que desde hacía 48 horas en la revisión ambulatoria se retiró el tratamiento con amiodarona y se sustituyó por $0.125 \mathrm{mg}$ de digoxina diario y un comprimido de atenolol $50 \mathrm{mg}$ tras observarse en el Holter episodios de flutter a $141 \mathrm{lpm}$ sintomáticos.

En la Unidad Coronaria presenta numerosos eventos arrítmicos del tipo de las taquicardias ventriculares y torsades des pointes en las 24 horas posteriores a su ingreso y posteriormente evoluciona favorablemente permitiendo a las 72 horas la retirada del marcapasos transitorio y el traslado a la planta de Cardiología donde se monitoriza con telemetría. Se observan varios episodios de flutter con respuesta ventricular rápida que se manejan con dosis ascendentes de betabloqueantes. Vuelve a presentar ritmo sinusal a $60 \mathrm{lpm}$ con prolongación de intervalo QT y un episodio autolimitado tipo torsades des pointes asintomático por lo que se reduce el tratamiento bradicardizante. Se implanta Holter subcutáneo y la paciente es dada de alta a los 10 días de su ingreso con tratamiento anticoagulante y con atenolol $75 \mathrm{mg}$ diarios.

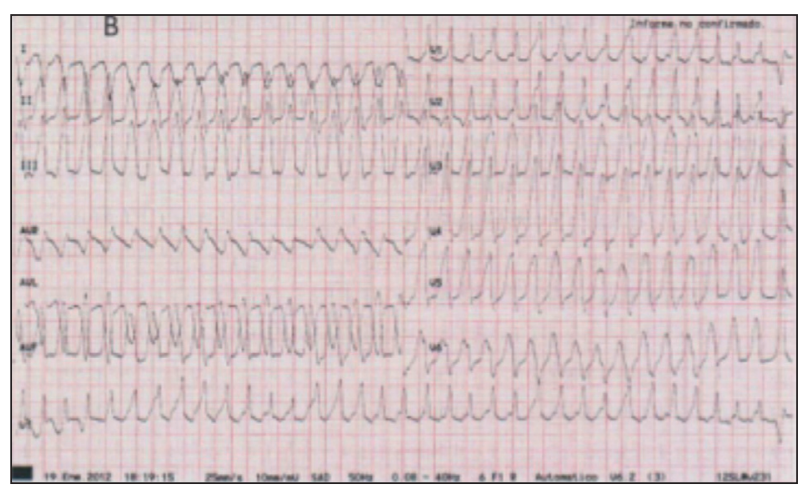

Figura 2B. Taquicardia ventricular con $Q R S$ ancho que precisó cardioversión eléctrica para su resolución. 


\section{Diagnóstico: Síndrome de QT Largo secundario a la asociación de medicación antiarrítmica}

El síndrome de QT largo se caracteriza por una grave alteración de la repolarización ventricular que se manifiesta electrocardiográficamente por la prolongación del intervalo QT corregido por la fórmula de $B a z e t t^{1} \geq$ de $440 \mathrm{~ms}$ en varones y $\geq 460 \mathrm{~ms}$ en mujeres. Las causas responsables pueden ser congénitas ${ }^{2} \mathrm{o}$ adquiridas. Estas últimas son las más frecuentes y entre ellas la farmacológica ocupa el primer lugar. Entre los fármacos responsables se encuentran fármacos enlentecedores del nodo como los betabloqueantes, la digoxina, algunos antimicrobianos como los macrólidos o las quinolonas ${ }^{3,4}$ y determinados fármacos psicótropos. Otros causas adquiridas pueden ser estructurales o metabólicas como los trastornos electrolíticos (hipopotasemia, hipomagnesemia, hipocalcemia..), tóxicos, dietas proteicas líquidas, trastornos endocrinos como hipotiroidismo, la anorexia nerviosa, feocromocitoma o carcinoma suprarrenal ${ }^{5}$. Las manifestaciones clínicas son muy variables: puede cursar de forma asintomática, presentar síncopes recurrentes, crisis convulsivas o incluso muerte súbita como primera manifestación. Dentro del amplio espectro clínico destaca en este síndrome la predisposición a arritmias ventriculares malignas del tipo torsades des pointes como las que padeció nuestra paciente.

Entre los factores de riesgo que favorecen este síndrome en nuestra paciente se encuentran el uso de al menos dos fármacos favorecedores, la hipertrofia ventricular izquierda, la presencia de insuficiencia cardíaca crónica y las reversiones a ritmo sinusal de FA paroxística.

Es conocido el efecto proarritmogénico de los fármacos antiarrítmicos. Nuestra paciente tomaba dos de ellos lo cual puede explicar la presencia de taquicardias supraventricu- lares, episodios de fibrilación auricular y flutter autolimitados. Además la presencia de estas arritmias mencionadas en el contexto de QT largo favorecen también la aparición de TVNS, TVSV tipo torsades des pointes. Otro factor llamativo en esta paciente es que tras la CVE se objetivó bradicardia extrema con un QTc muy largo (no disponemos de ECG), probablemente por la asociación de atenolol y digoxina. Esta situación se ve con relativa frecuencia en pacientes que acuden para realización de CVE programada y se encuentran en tratamiento con atenolol, digoxina y amiodarona.

En este caso en concreto se consideró la causa responsable la asociación de fármacos antiarrítmicos con el predisponente de arritmias supraventriculares paroxísticas.

En revisiones posteriores la paciente se ha mantenido asintomática desde el punto de vista cardiovascular y en estudios con Holter solo ha presentado rachas de taquicardia supraventricular autolimitada con respuesta ventricular media en torno a 105-115 1pm.

\section{BIBLIOGRAFÍA}

1. Bazett H. An analysis of the time-relations of electrocardiograms. Heart.1920; 7: 353-70.

2. Medeiros-Domingo A, Iturralde-Torres P, Ackerman M. Clínica y genética en el síndrome de QT largo. Rev Esp Cardiol 2007; 60 (7):739-52.

3. Salinas Arce J, Romero R, Solórzano P. Un caso de QT largo y torsades des pointes por ciprofloxacino. Rev Esp Cardiol 2010; 63: 111-2.

4. Ridruejo R, Zalba B, Civeira E, Montes N, Munárriz J. Síndrome de QT largo adquirido en pacientes ingresados en UCI. Med Intensiva 2005;29(7):379-83.

5. García L, Couto D, Rodriguez JA. Una extraña etiología para el QT largo. Rev Esp Cardiol 2012;65:479-488. 